\title{
Assisted Interpretation of Laser-Induced Fluorescence Spectra of Egg-Based Binding Media Using Total Emission Fluorescence Spectroscopy
}

\author{
Austin Nevin ${ }^{1,2}$ and Demetrios Anglos ${ }^{1}$ \\ ${ }^{1}$ Institute of Electronic Structure and Laser, Foundation for Research and Technology-Hellas (IESL-FORTH), \\ P.O. Box 1527, 71110 Heraklion, Greece \\ ${ }^{2}$ Courtauld Institute of Art, Somerset House, Strand, London WC2R ORN, UK
}

Received 13 September 2006; Revised 20 October 2006; Accepted 27 October 2006

Recommended by Marta Castillejo

Laser-induced fluorescence (LIF) spectroscopy can provide nondestructive, qualitative analysis of protein-based binding media found in artworks. Fluorescence emissions from proteins in egg yolk and egg white are due to autofluorescent aromatic amino acids as well as other native and age-related fluorophores, but the potential of fluorescence spectroscopy for the differentiation between binding media is dependent on the choice of a suitable excitation wavelength and limited by problems in interpretation. However, a better understanding of emission spectra associated with LIF can be achieved following comparisons with total emission fluorescence spectra where a series of consecutive emission spectra are recorded over a specific range. Results using nanosecond UV laser sources for LIF of egg-based binding media are presented which are rationalised following comparisons with total emission spectra. Specifically, fluorescence is assigned to tryptophan and oxidation products of amino acids; in the case of egg yolk, fatty-acid polymerisation and age-related degradation products account for the formation of fluorophores.

Copyright (c) 2006 A. Nevin and D. Anglos. This is an open access article distributed under the Creative Commons Attribution License, which permits unrestricted use, distribution, and reproduction in any medium, provided the original work is properly cited.

\section{INTRODUCTION}

The analysis and understanding of painting materials is central to conservation, and often requires determination of the binding medium. Paintings often consist of multiple layers of pigments, colourants, and dyes which are typically dispersed in a matrix (binding medium) applied over a substrate and are often coated (varnished). Protein-based materials including egg white and egg yolk have traditionally been used as binding media and in conservation treatments; their identification can be critical for conservation, is of art historical interest, and is a challenge to the conservation scientist. However, analysis of proteins and other binding media typically involves taking samples so that characterisation using noninvasive methods is clearly advantageous due to the limitations associated with sampling of paintings. Recent advances in laser-induced fluorescence (LIF) spectroscopy have demonstrated a potential alternative nondestructive, qualitative means of investigating and analysing materials found in works of art; the interpretation of LIF spectra aided by information from total emission spectra is the focus of this article.

Fluorescence spectroscopy is widely applied for protein analysis in many fields, due to intrinsic fluorescence from aromatic amino acids tryptophan, tyrosine, and phenylalanine $[1,2]$. Especially sensitive to changes in the structure and environment, fluorescence emissions of amino acids are often used to follow dynamic reactions of known molecules in solution rather than for the identification of specific materials or proteins. In addition to amino acid fluorescence, a large range of fluorescent degradation products from aged proteins have been identified [3]. These include both photooxidation products from the combination and modifications of amino acids and cross-linkage reactions between amino acids and other compounds (e.g., sugars). For instance, the formation of dityrosine, which can occur both at the ortho- and para- positions, has been documented both with free amino acids [4] and in solid protein films [5].

However, fluorescence spectroscopy, including LIF, has not had widespread applications in the analysis of paintings, 
and specifically binding media. Initial research using spectrofluorimetry on artists' materials focused on the fluorescence of films of oil-based media [6] as well as on pigments bound in various media $[7,8]$. Investigations of LIF for the analysis of protein-based binding media have been limited to few instances; for example, LIF has been used as a monitoring tool for the laser cleaning of egg tempera [9].

One problematic aspect of fluorescence analysis of proteins, especially in mixtures, is the interpretation of fluorescence spectra. This is due to characteristically broad signals found with macromolecular fluorescence, and to the lack of investigations of the fluorescence properties of complex mixtures of the proteins found in works of art. Fluorescence databases or standards of binding media are not available, and single excitation spectra alone do not always provide sufficient diagnostic information for the determination of the origin of protein-based films. However, studies on analogous samples of proteins from food have demonstrated the potential of total emission spectroscopy for discrimination between complex multiple protein [10] and multiple oil-based systems [11]. Total emission spectroscopy, although more time-consuming to record than a single emission spectrum, can provide more complete information, especially regarding the presence of fluorophores, their wavelength dependence, their emission and excitation maxima, and the relationship between multiple fluorophores.

The aim of this work is to investigate the potential of total emission spectroscopy combined with LIF for the assessment of the fluorophores present in egg white and egg yolk films.

\section{EXPERIMENTAL}

Following investigations of LIF to identify and discriminate among protein samples of binding media found in works of art using various excitation sources [2], further analysis of films of egg-based binding media was carried using total emission spectroscopy. Although the major proteinaceous components of both egg yolk and egg white are the same (ovalbumin and lysozyme), egg yolk also contains significant fatty acids (including palmitic and oleic acid).

\section{METHODOLOGY AND METHODS}

Egg white and egg yolk used as binding media were prepared as indicated in artists' accounts and historical recipes [12]. Egg white was beaten to form stiff peaks and left for 24 hours; foam was skimmed and removed and a solution of $50 \% \mathrm{w} / \mathrm{w}$ clear egg white in nanopure water was prepared. Egg yolk was extracted from egg by piercing the yolk and allowing the liquid to drip from the encasing film; the yolk was diluted in nanopure water to give a 50\% w/w emulsion. Films of proteins were cast on polished fused silica disks to give film thickness of approximately $15 \mu \mathrm{m}$ (perthometer S5P profilometer) and were examined 1 year after their preparation.

Laser-induced fluorescence of films of proteins was recorded using $248 \mathrm{~nm}$ excitation from a $\mathrm{KrF}$ excimer laser (TUI Laser AG BraggStar 200), 10 ns pulse duration. A max-

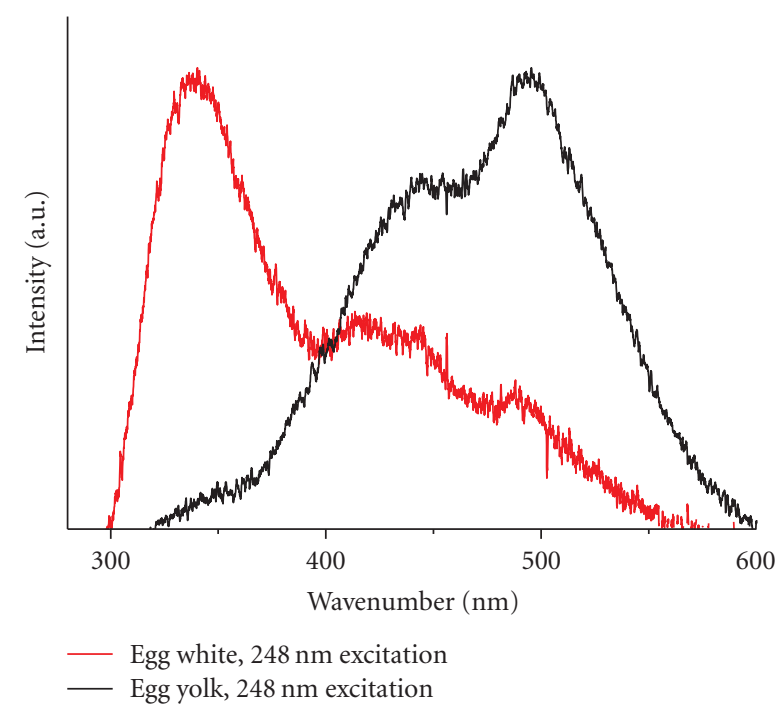

FIGURE 1: LIF spectra of egg white and egg yolk at the excitation wavelength of $248 \mathrm{~nm}$ ( $\mathrm{KrF}$ excimer laser, $10 \mathrm{~ns}$ excitation).

imum of 500 pulses at a fluence $5 \mathrm{mJcm}^{-2}$ per pulse with a spot size $1 \times 1 \mathrm{~mm}$ was used when recording spectra. Spectra were also recorded using the $3 \mathrm{rd}$ harmonic $(355 \mathrm{~nm}$ ) of a Q-switched Nd : YAG (Spectron Laser Systems), 10 ns pulse duration, a maximum of 300 pulses, and fluence $5 \mathrm{~mJ} \mathrm{~cm}^{-2}$ per pulse. LIF emission was collected using a fibre-optic placed at approximately $60^{\circ}$ to the sample axis coupled to an Ocean Optics HD4000 spectrophotometer with approximately $0.13 \mathrm{~nm}$ resolution. In addition, films were analysed using a scanning spectrofluorimeter with excitation provided by a Xenon arc lamp (Jobin-Yvon/Horiba Fluoromax-P). Typical scanning parameters were integration time of $0.2 \mathrm{~s}$ per point, intervals of $1 \mathrm{~nm}$, and excitation/emission slits between $1-5 \mathrm{~nm}$. Films of proteins were analysed at $30^{\circ}$ from the sample-axis. Total emission spectra were constructed by recording a series of excitation spectra with an interval of $5 \mathrm{~nm}$ between successive excitation scans and using a resolution of $1 \mathrm{~nm}$ emission spectra; scans were recorded with excitation from $220 \mathrm{~nm}$ to $400 \mathrm{~nm}$, and first- and second-order Rayleigh scattering was eliminated.

\section{RESULTS AND DISCUSSION}

For clarity, results of LIF spectral analysis on model films are presented first, and these are followed by detailed spectrofluorimetric analysis using total emission spectroscopy undertaken to characterise the emission pattern of each mixture.

\subsection{Laser-induced fluorescence of films}

Autofluorescence of proteins seen in LIF spectra of egg white (Figure 1) is due to the presence of aromatic amino acids tryptophan, tyrosine, and phenylalanine, which account for fluorescence emissions between approximately $280 \mathrm{~nm}-$ $380 \mathrm{~nm}[1,2]$. 


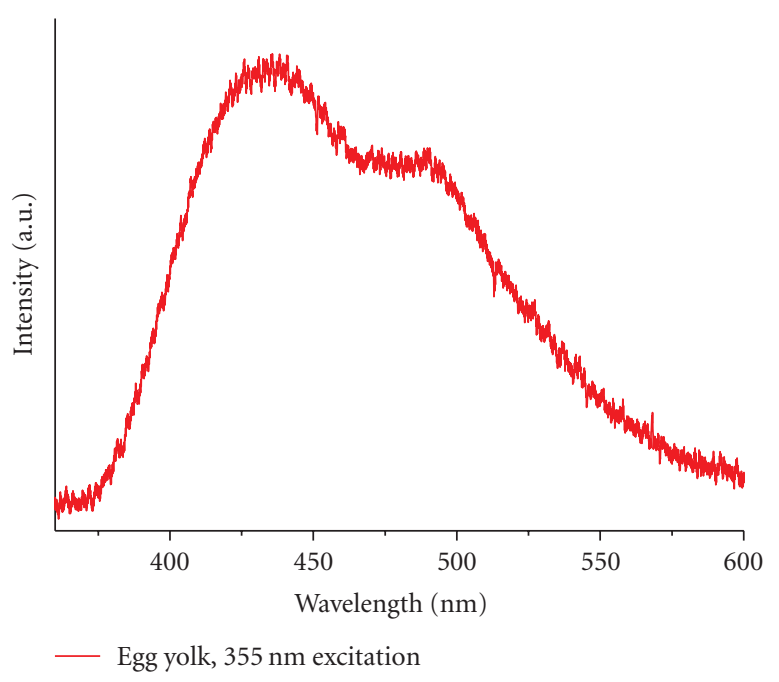

FIGURE 2: LIF spectra of film of egg yolk at the excitation wavelength of $355 \mathrm{~nm}$ (3rd harmonic of Nd : YAG 3rd laser, $10 \mathrm{~ns}$ ).

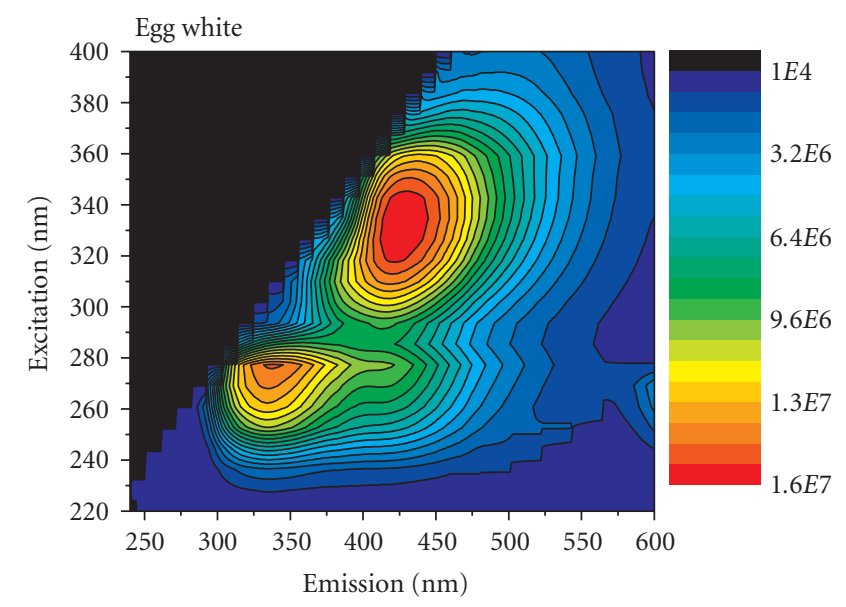

FIgURE 3: Total emission spectrum of a film of egg white, right scale counts, with intervals of $3 \times 10^{6}$ counts between each line/colour.

Further emissions at wavelengths greater than $380 \mathrm{~nm}$ are especially apparent in egg yolk (Figure 1). In addition, a strong emission from egg yolk following excitation at $355 \mathrm{~nm}$ is observed (Figure 2). However, fluorescence at wavelengths greater than $380 \mathrm{~nm}$ cannot be ascribed to amino acid side chains.

\subsection{Total emission spectroscopy}

Spectrofluorimetric analysis of samples of egg white films to produce total emission spectra yields significantly different results for egg white and egg yolk, as seen in Figures 3 and 4.

Examination of total emission spectra immediately allows the location of emission maxima and indicates the presence of multiple fluorophores and the dependence of the emission on excitation. Clear differences in the spectral patterns of egg white and egg yolk are visible; the former is as-

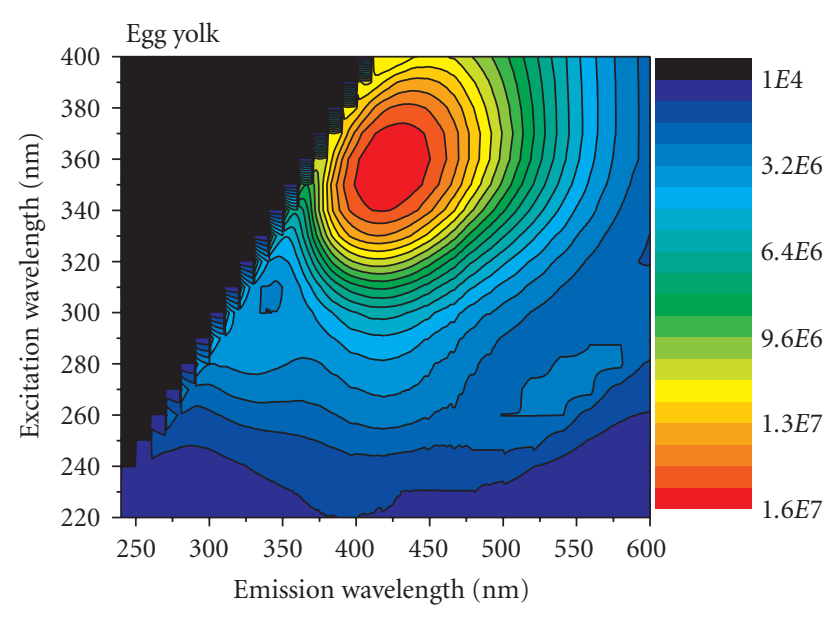

FIGURE 4: Total emission spectrum of a film of egg yolk, right scale counts, with intervals of $3 \times 10^{6}$ counts between each line/colour.

sociated with multiple and relatively sharp emissions, and the latter with a single-broad emission. Both media exhibit a maximum emission at approximately $425 \mathrm{~nm}$ (excitation maximum at $355 \mathrm{~nm}$ ), which can be ascribed to the accumulation of photodegration products of egg proteins and other compounds present in the complex mixtures found within eggs.

Degradation can result from photooxidation of amino acids and of other compounds found within eggs (including flavins, free glucose and, lipids (in egg yolk)), or from cross-linkage reactions between amino acids and sugars [13]. While collagen-based materials used in binding media are characterised by fluorophores which develop following the Maillard reaction [14], uncooked and untreated egg proteins used as binding media, which have been reported to form Maillard reaction products [15], do not exhibit characteristic fluorescence profiles at $380 \mathrm{~nm} \mathrm{[2].} \mathrm{Rather,} \mathrm{the} \mathrm{formation}$ of dityrosine [16], suggested in the case of photooxidised collagen-based binding media [17], and tryptophan oxidation products $\mathrm{N}$-formylkynurenine (NFK) and kynurenine (associated with emissions at approximately $435 \mathrm{~nm}$ ) [18] may further account for observed emissions.

\subsection{Interpretation of total emission spectra}

In the total emission spectra, differences in the shape of the excitation-emission maxima and the contour lines surrounding the maximum suggest the presence of more than one fluorophore, especially in the case of films of egg white, which likely contains oxidised tyrosine (emission maximum at approximately $410 \mathrm{~nm}$ ). In egg white, other fluorescence emissions observed at $335 \mathrm{~nm}$ (excitation maximum at $290 \mathrm{~nm}$ ) are likely due to native tryptophan; contributions from tyrosine can also be inferred from the emission at approximately 300-320 nm. Importantly, no similar emissions are found in egg yolk films. The assignment of fluorescence to photooxidation products of tyrosine degradation is based on 


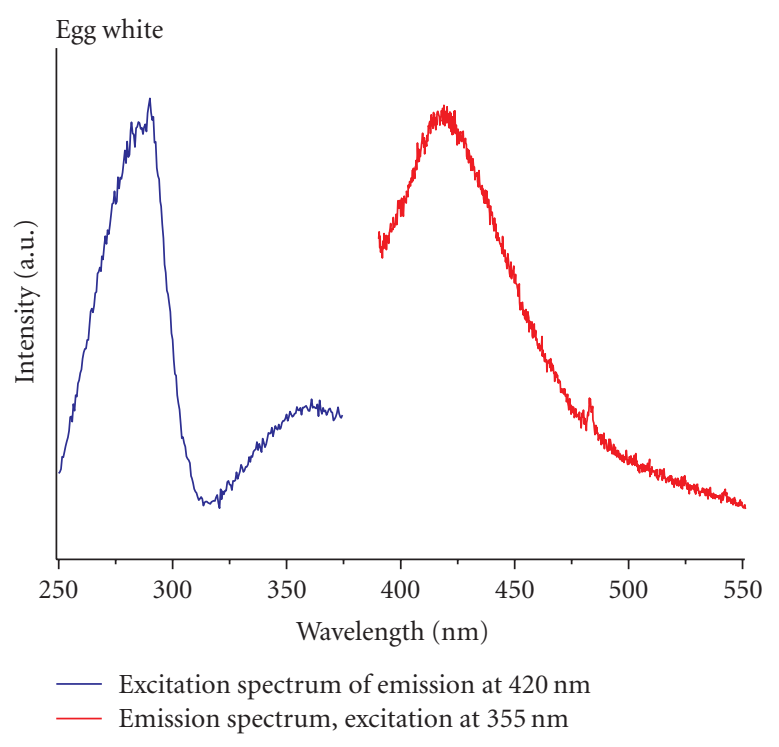

FIgURE 5: Excitation spectrum (blue) (maxima at 285 and $355 \mathrm{~nm}$ ) and emission spectrum (red) (excitation at $355 \mathrm{~nm}$ ) of a film of egg white.

the characteristic excitation spectrum of dityrosine (emission maximum of $410 \mathrm{~nm}$, excitation maxima at 285 and $355 \mathrm{~nm}$ ) [19] seen in Figure 5.

Importantly, the assignment of emissions observed in egg proteins to dityrosine and oxidised tryptophan based on florescence data alone can be misleading, due to additional fluorophores which have been documented to fluoresce in the same range [20]. Nonetheless, total emission spectra allow a greater understanding of the fluorphores found in single-wavelength excitation, as in LIF.

\subsection{Interpretation of LIF spectra}

Therefore, a possible interpretation of the LIF spectra of egg yolk (Figure 3) is that fluorescence observed at $425 \mathrm{~nm}$ is a combination of emission from dityrosine (emission $410 \mathrm{~nm}$ ), NFK (emission $435 \mathrm{~nm}$ ), and DOPA (emission at $480 \mathrm{~nm}$ ), as well as potential contributions from riboflavin (emission between $500-550 \mathrm{~nm}$ ) [21] and flavin photooxidation products (lumichrome and lumiflavin); fatty-acid polimerisation could result in the production of new flourophores. Additionally, the generation of photooxidation products may be induced by sensitisation of riboflavin by visible radiation, present in both egg white and egg yolk [22].

\section{CONCLUSIONS}

Results demonstrate that LIF can be used to distinguish between proteins in art works, and that emissions have a strong wavelength dependency; the fluorescence "fingerprint" or total emission spectrum can greatly assist the choice of laser wavelength for LIF spectroscopy as well as provide more information regarding the fluorophores present in complex mixtures. In this case, examination of the total emission spectra of egg yolk and egg white immediately allows their separation and provides a useful tool for the interpretation of LIF spectra.

Within the context of organic analysis in conservation, LIF is advantageous as it is a noninvasive, nondestructive, simple, and rapid technique which can be used to distinguish between different proteins, provided a difference in fluorescence is apparent. Due to the identification of multiple fluorophores in the complex protein mixtures found in binding media and seen in total emission spectra, techniques capable of better separating fluorescence contributions would be useful. In addition, changes in the fluorescence emission spectra as a result of ageing should be investigated. Finally, future work should focus on the ageing of protein media, pigmentmedia interactions, and investigations of fluorescence lifetime.

\section{ACKNOWLEDGMENT}

The first author's research was supported by the European Union 6th Framework Programme Marie Curie Early Stage Training Fellowship as part of the ATHENA Project (MESTCT-2004-504067).

\section{REFERENCES}

[1] A. S. Ladokhin, "Fluorescence spectroscopy in peptide and protein analysis," in Encyclopedia of Analytical Chemistry, R. Meyers, Ed., pp. 5762-5779, John Wiley \& Sons, New York, NY, USA, 2000.

[2] A. Nevin, S. Cather, D. Anglos, and C. Fotakis, "Analysis of protein-based binding media found in paintings using laser induced fluorescence spectroscopy," Analytica Chimica Acta, vol. 573-574, pp. 341-346, 2006.

[3] Z. Deyl, I. Mikšík, and J. Zicha, "Multicomponent analysis by off-line combination of synchronous fluorescence spectroscopy and capillary electrophoresis of collagen glycation adducts," Journal of Chromatography A, vol. 836, no. 1, pp. 161-171, 1999.

[4] W. G. Liu, K. D. Yao, G. C. Wang, and H. Li, "Intrinsic fluorescence investigation on the change in conformation of crosslinked gelatin gel during volume phase transition," Polymer, vol. 41, no. 20, pp. 7589-7592, 2000.

[5] M. Lacroix, T. C. Le, B. Ouattara, et al., "Use of $\gamma$-irradiation to produce films from whey, casein and soya proteins: structure and functionals characteristics," Radiation Physics and Chemistry, vol. 63, no. 3-6, pp. 827-832, 2002.

[6] E. R. de la Rie, "Fluorescence of paint and varnish layers (part I)," Studies in Conservation, vol. 27, no. 1, pp. 1-7, 1982.

[7] T. Miyoshi, "Fluorescence from pigments in fresh and stored oil colours under $\mathrm{N}_{2}$ laser excitation," Japanese Journal of Applied Physics, vol. 24, no. 8, pp. 1113-1114, 1985.

[8] T. Miyoshi, "Fluorescence from Resins for oil painting under $\mathrm{N}_{2}$ laser excitation," Japanese Journal of Applied Physics, vol. 29, pp. 1727-1728, 1990.

[9] M. Castillejo, M. Martín, M. Oujja, et al., "Evaluation of the chemical and physical changes induced by $\mathrm{KrF}$ laser irradiation of tempera paints," Journal of Cultural Heritage, vol. 4, suppl. 1, pp. 257s-263s, 2003.

[10] C. M. Andersen and R. Bro, "Practical aspects of parafac modeling of fluorescence excitation-emission data," Journal of Chemometrics, vol. 17, no. 4, pp. 200-215, 2003. 
[11] E. Sikorska, T. Górecki, I. V. Khmelinskii, M. Sikorski, and J. Kozioł, "Classification of edible oils using synchronous scanning fluorescence spectroscopy," Food Chemistry, vol. 89, no. 2, pp. 217-225, 2005.

[12] C. Cennino D'Andrea, Il libro dell'Arte, Dover, New York, NY, USA, 1960.

[13] M. J. Davies, S. Fu, H. Wang, and R. T. Dean, "Stable markers of oxidant damage to proteins and their application in the study of human disease," Free Radical Biology and Medicine, vol. 27, no. 11-12, pp. 1151-1163, 1999.

[14] D. G. Dyer, J. A. Blackledge, S. R. Thorpe, and J. W. Baynes, "Formation of pentosidine during nonenzymatic browning of proteins by glucose: identification of glucose and other carbohydrates as possible precursors of pentosidine in vivo," Journal of Biological Chemistry, vol. 266, no. 18, pp. 11654-11660, 1991.

[15] L. Campbell, V. Raikos, and S. R. Euston, "Modification of functional properties of egg-white proteins," Nahrung - Food, vol. 47, no. 6, pp. 369-376, 2003.

[16] C. Giulivi, N. J. Traaseth, and K. J. A. Davies, "Tyrosine oxidation products: analysis and biological relevance," Amino Acids, vol. 25, no. 3-4, pp. 227-232, 2003.

[17] M. Oujja, E. Rebollar, C. Abrusci, A. Del Amo, F. Catalina, and M. Castillejo, "UV, visible and IR laser interaction with gelatine," to appear in Journal of Applied Physics: Conference Series.

[18] J. L. E. Reubsaet, J. H. Beijnen, A. Bult, R. J. van Maanen, J. A. D. Marchal, and W. J. M. Underberg, "Analytical techniques used to study the degradation of proteins and peptides: chemical instability," Journal of Pharmaceutical and Biomedical Analysis, vol. 17, no. 6-7, pp. 955-978, 1998.

[19] A. J. Kungl, M. Breitenbach, and H. F. Kauffmann, "Molecular dynamics simulation of the rare amino acid LL-dityrosine and a dityrosine-containing peptide: comparison with timeresolved fluorescence," Biochimica et Biophysica Acta-General Subjects, vol. 1201, no. 3, pp. 345-352, 1994.

[20] P. Guptasarma and D. Balasubramanian, "Dityrosine formation in the proteins of the eye lens," Current Eye Research, vol. 11, no. 11, pp. 1121-1125, 1992.

[21] S. Hustad, P. M. Ueland, and J. Schneede, "Quantification of riboflavin, flavin mononucleotide, and flavin adenine dinucleotide in human plasma by capillary electrophoresis and laser-induced fluorescence detection," Clinical Chemistry, vol. 45, no. 6 pt 1, pp. 862-868, 1999.

[22] A. M. Edwards and E. Silva, "Effect of visible light on selected enzymes, vitamins and amino acids," Journal of Photochemistry and Photobiology B: Biology, vol. 63, no. 1-3, pp. 126-131, 2001. 